\title{
DEVELOPMENT OF A BIM LIBRARY FOR BUILDING ENERGY RETROFITTING: CURRENT WORKFLOWS AND A PROPOSAL FOR A RECOMMENDER SYSTEM
}

\author{
Angelo L.C. Ciribini ${ }^{1}$, Michele Melchiori ${ }^{2}$, Lavinia C. Tagliabue ${ }^{1}$, and Silvia Mastrolembo Ventura ${ }^{1}$ \\ ${ }^{1}$ University of Brescia, Department of Civil, Environmental, Architectural Engineering and Mathematics, \\ Brescia, Italy \\ ${ }^{2}$ University of Brescia, Department of Information Engineering, Brescia, Italy
}

\begin{abstract}
Building Information Modeling (BIM) is an objectoriented design process based on the assembling of building components virtually prototyped. Both the BIM objects available in BIM authoring platforms and the ones provided by manufacturers in digital marketplaces do not include the level of information need suitable to manage each purpose and use case within a BIM environment. Considering the purpose of energy retrofitting, this study analyzes the use of a BIM library of External Thermal Insulation Composite Systems (ETICS) as provided by a global manufacturer. The set of requirements for supporting a preliminary energy analysis is evaluated. Moreover, a data spreadsheet for energy analysis, bi-directionally linked to the BIM model, has been developed as a calculation tool for the energy evaluation of design options. Finally, the outline concept of a web-based system able to support the recommended selection of the most suitable BIM object from one or more BIM libraries based on various project parameters, including the selected BIM uses, is proposed.
\end{abstract}

\section{Introduction}

\section{Object-oriented design and BIM libraries}

BIM-oriented design is an information modeling and management process based on the assembling of building components virtually prototyped (i.e., BIM objects) (Hjelseth, 2011). Building Information Modeling (BIM), in fact, could be defined as "the object-oriented modeling of the data-rich building components, incorporating 3D geometries, spatial information, thermal values, and material properties; parameters upon which data interoperability builds up" (Gourlis \& Kovacic, 2017). Moreover, data interoperability could be defined as the ability of BIM authoring platforms and BIM tools to exchange data and operate on them within a BIM environment (Eastman et al., 2011) based on specific purposes, uses and related workflows.

A BIM use is a "method of applying building information modeling during the lifecycle of a facility" in order to "achieve one or more specific objectives"
(Kreider \& Messner, 2013). The level of information need to be embedded within a BIM object represents the degree of reliability regarding the implementation of BIM processes and technologies suitable for specific use cases (Kreider \& Messner, 2013). Relevant information content in BIM objects should be therefore managed according to specific BIM uses in order to exchange consistent, relevant, and reliable information (Hjelseth, 2011).

When dealing with parametric and object-oriented modeling, object classes (e.g., walls, doors, slabs, and so forth) define how the object instances "are structured, how they are edited and how they behave when their context changes" (Sacks et al., 2018). A library of BIM objects is, therefore, a set of object classes, which can be provided as either predefined by each BIM authoring platform or user-defined to create customized digital representation of building components. The latter option could be adopted by the users of BIM authoring platforms in order to manage some limitations of predefined BIM objects in relation, for instance, to the level of information need of each BIM object based on the relevant use cases. This capability is considered as an essential skill for "any company or organization which appears as BIMaware" in order to use them as effective knowledge containers on a routine basis (Sacks et al., 2018).

BIM objects are also available in web-based marketplaces (e.g., BIMobject, MagiCAD Cloud, MEPcontent, etc.), where manufacturers can provide their own building components to be used in BIMbased projects. Most of the digital marketplaces allows downloading BIM objects that are compatible with Autodesk Revit. Sketch Up, ArchiCAD, Allplan and Vectorworks, for example, are other BIM authoring platforms for which BIM objects are also available in digital marketplaces, although in small numbers compared to those available for Revit. In some cases, manufacturers also allow users to download the Industry Foundation Classes (IFC) version of BIM objects (e.g., 17.100 out of 61.686 object families are available in the IFC format in BIMobject $\left.{ }^{\circledR}\right)$. Some plug-ins also exist for BIM authoring platforms in order to download BIM objects 
directly within the BIM authoring platforms from digital marketplaces.

\section{The management of the BIM objects for energy- related applications}

Both the BIM objects available in BIM authoring platforms and the ones provided by manufacturers in digital marketplaces do not include the level of information need suitable to manage each purpose and use case within a BIM environment. In order to narrow the scope of the study down to one possible BIM use for the design phase of a building facility, energy retrofitting has been taken into account.

In the energy modeling process, object relationships and semantics related to architectural models do not substantially affect simulation results and they are often abstracted, risking delays in the result interpretation over the design stage (Kim et al, 2016). Consistent object classifications and semantics between BIM and BEM (Building Energy Modeling) are needed to guarantee interoperable BIM-to-BEM data flows and design processes. For this reason, if the energy analysis represents a BIM use, the building components managed as BIM objects should contain the parameters that are required to enable energy analysis-oriented tools receiving values from the BIM model. Consequently, data exchanges between the building objects and their attributes between different domains could be managed more effectively and could guarantee reliable results from the analysis.

\section{BIM libraries-based recommendation}

As already said, predefined BIM objects could not be used for any purposes and uses; user-defined attributes are needed for specific applications, indeed. Moreover, within the digital marketplaces, BIM objects can be searched in by categories and file formats; no information is usually available neither about their informative content nor the specification for the BIM uses they can be implemented for. It could be useful, instead, to guide users in the selection of the most appropriate BIM object based on their needs. For that reason, this study also proposes a novel recommender system as a method to guide the users in the selection of the most appropriate BIM object based on BIM purposes and use cases.

In fact, similarly to what happens for e-commerce business models, a recommender system permits to deliver context-aware suggestions to the design teams in order to reduce their effort in manually searching the most appropriate BIM objects into one or more libraries building components virtually prototyped. Recommender systems, also known as recommendation systems, aim to suggest to the shoppers (i.e., the practitioners) those items that are most probably of interest for them among the ones available in a repository. As well as they can reduce the time and effort required to a user in making a choice, for example discovering a recent movie to be watched related with the user's concerns (Ricci et al., 2015). Recommenders, in fact, are used in an integrated way as sources of knowledge in order to infer users' interests. The most common type of knowledge lies with the users' feedbacks, which can be explicit or implicit. An example of explicit feedback is the rating a user can provide for an item she/he purchased. Moreover, the simple act of buying an item or accessing to its description can be interpreted as an endorsement for the item and, therefore, implicit feedback for it. The typical problem solved by recommenders is thus the prediction of the level of interest, or rating, a user will assign to a given item. However, for the largest portion of the practical applications, it can be sufficing to identify and propose as ranked list the top-k relevant items for a specific user (Aggarwal, 2016).

Several recommendation techniques have been developed. For example, the collaborative filtering technique exploits choices or ratings of users that are similar to a user A, or made similar past choices, in order to establish unknown interests of A. This technique suffers from the "cold start problem", where items with no evaluations are ruled out by recommendations. In content-based techniques, descriptive attributes of items are considered to suggest items to a user that are similar to items rated with a high score by the same user. Hybrid techniques exploit the availability of different information sources to apply and combine collaborative contents, as well as social and context-aware recommendation models also in order to overcome the weaknesses of the single model techniques (Burke, 2007).

\section{Structure of the paper and scope of the study}

Based on the background described in the previous paragraphs, the paper is organized as follows. In the next paragraph, as an example, the customization of a manufacturer's BIM library for supporting energyrelated analysis in energy retrofitting design processes is described. In particular, this example application is related to the energy calculations for a retrofitting project applied to three types of buildings. According to the Italian national regulation, the level of information need to consider for such a use case is identified.

Furthermore, the development of a data spreadsheet bi-directionally linked to the building information model for the preliminary energy-related analysis of design options has been developed for each type of building. Then, as a result of this example application, the concept of a recommender system, which could help the users in selecting the most appropriate BIM objects for the specific use case from one or more BIM libraries has been proposed. In this study, the focus stays with the application of the recommender system for energy retrofitting. However, this study represents 
a first step into the development of web systems able to support users in the selection of the most appropriate BIM object from one or more BIM libraries based on various project parameters, related to BIM purposes and use cases.

\section{Development of a BIM library for the BEM use case}

\section{Research method and materials}

Three building typologies have been used as a test field to double check the energy performance achieved through standardized envelope components suitable for energy retrofit. The BIM models of the buildings have been realized and BIM objects have been used to develop a BIM library to test the different solution by a bi-directional link (i.e., through Visual Programming Language VPL) between the BIM model and an Excel spreadsheet, where equations to calculate the energy parameters and indicators included in the national regulation have been implemented. In this way, the energy performance deriving from each configuration could be tested and compared to support the decisionmaking process. From the BIM model, the geometrical information and the energy parameters associated to the materials adopted for the components' layers are transferred through VPL scripts to the Excel spreadsheet. The same parameters will be included in the recommender system to filter the BIM objects most suitable to achieve energy goals. The building performance is not only due to components performance but also to the building configuration (e.g., shape, complexity, the ratio between opaque and transparent envelope, etc.); for that reason, the test cases have been developed for the energy comparison of the baseline (i.e., existing situation) and the design case (i.e., retrofitted building).

\section{Case studies and BIM models}

The BIM models of the three case studies (i.e., buildings with different destinations and characteristics) have been realized in Autodesk Revit. They are: (a) a single family house; (b) a multi-story residential building; (c) an office building (Figure 1).
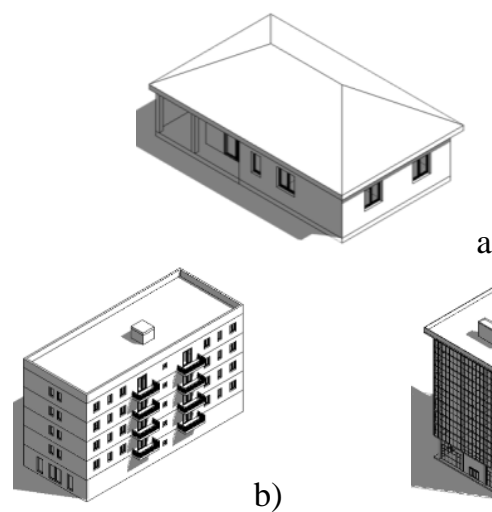

a)

b)

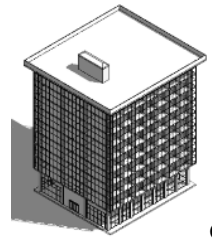

c)

Figure 1: Typologies of buildings adopted as case studies
The building (a) is a $100 \mathrm{~m}^{2}$ single family house with three bedrooms, two bathrooms and a kitchen-cumliving room. This is a commonly adopted typology, widespread throughout the national territory. Consequently, the energy retrofitting of this type of building has wide energy and economic potential considering the lifespan of the building and the age of the existing building stock. The building (b) is multistory residential building with 16 apartments of about $80 \mathrm{~m}^{2}$ each, with a kitchen-cum-living room, two bedrooms, a bathroom and a storeroom. The building (c) is an office with a very different widely glazed envelope; the vertical distribution is concentrated in the core, where stairs and elevators are located. The floor is $600 \mathrm{~m}^{2}$ and the height is $35 \mathrm{~m}$ (i.e., $6 \mathrm{~m}$ for the ground floor and $3.5 \mathrm{~m}$ for the type floor).

The case studies have been tested with the BIM objects products and components (i.e. mainly opaque for the residential buildings a) and b) and glazing systems for the office building $\mathrm{c}$ ). The thermal properties of the case studies replicate the characteristics of the existing residential national built environment, with no insulation layer in the opaque envelope and installing single glazed windows. The buildings have been tested in three representative Italian climate locations (Table $1)$.

Table 1: Information about the three locations

\begin{tabular}{lccc}
\hline Info & Milan & Rome & Palermo \\
\hline Latitude & $45.4773 \mathrm{~N}$ & $41.9109 \mathrm{~N}$ & $38.1121 \mathrm{~N}$ \\
Longitude & $9.1815 \mathrm{E}$ & $12.4818 \mathrm{E}$ & $13.3366 \mathrm{E}$ \\
Cl. zone & $\mathrm{E}$ & $\mathrm{D}$ & $\mathrm{B}$ \\
DD & 2404 & 1415 & 751 \\
HDays & 182 & 166 & 121 \\
\hline
\end{tabular}

\section{Existing envelope conditions}

The existing envelope conditions are described in Table 2 .

Table 2: Existing envelope in the BIM model

\begin{tabular}{|c|c|}
\hline Vertical wall & Thickness [mm] \\
\hline - $\quad$ lime and cement plaster & 15 \\
\hline - hollow brick & 250 \\
\hline - lime and cement plaster & 15 \\
\hline Ground floor & Thickness [mm] \\
\hline $\begin{array}{l}\text { expanded clay concrete for } \\
\text { unventilated substrates }\end{array}$ & \\
\hline - reinforced concrete & 200 \\
\hline - $\quad$ sand and cement screed & 40 \\
\hline $\begin{array}{l}\text { - waterproofing membrane } \\
\text { - ceramic tiles }\end{array}$ & 10 \\
\hline Ceiling & Thickness [mm] \\
\hline - lime and cement plaster & 15 \\
\hline - hollow-core concrete slab & 240 \\
\hline - $\quad$ sand and cement screed & 40 \\
\hline Roof & Thickness [mm] \\
\hline - lime and gypsum plaster & 15 \\
\hline - hollow-core concrete slab & 240 \\
\hline - $\quad$ sand and cement screed & 40 \\
\hline
\end{tabular}


The existing envelopes for the three buildings have been retrofitted using the selected External Thermal Insulation Composite System (ETICS) as described in the following sections. The BIM models have been equipped with the BIM objects provided by a global manufacturer and customizing the thickness of the insulation layers to accomplish the required performances in the different climates (Table 1).

\section{Energy information modeling supported by a BIM library}

The BIM libraries created by the aforementioned manufacturer provides useful datasets to characterize the transparent or opaque envelope. The BIM library of an ETICS, freely downloadable from the manufacturer's website, has been adopted in order to conceive some retrofitting measures to enhance the energy quality of the existing buildings (a), (b) and (c). Moreover, the characteristics included in the BIM objects have been analyzed in order to understand their suitability and reliability to support the energy analysis. The aim is to compare the existing situation and the retrofitted one on a performance basis (Figure 2). Predefined object classes and set of requirements have been used to create the BIM base case.

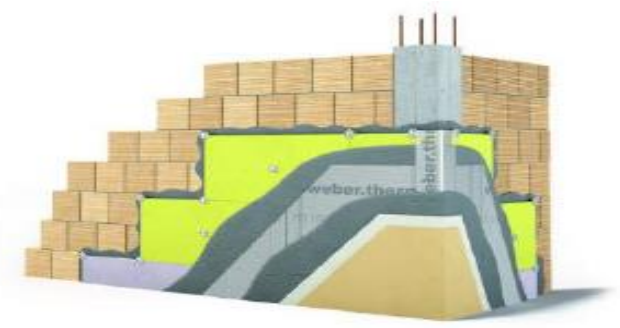

Figure 2: ETICS adopted as retrofit system

The system has been selected taking into account the market penetration as well as the energy and environmental performances (i.e., rock wool with $80 \%$ of recycled material).

BIM object for the energy retrofitting of the opaque vertical and horizontal envelope

The manufacturer's BIM library generates and provides a sample file with specific geometrical (Table 3; Figure 3), thermal and acoustic performances. Design flexibility is endorsed to define specified performance system's profiles. In the "type properties" (Figure 2) the main system's features have been reported; in some cases, information can be defined through the materials' attributes (e.g., total thickness: $393.5 \mathrm{~mm}$, thermal transmittance (declared): 0.25 $\mathrm{W} / \mathrm{m}^{2} \mathrm{~K}$ ), while for other specific parameters it has been specified by means of laboratory tests considering the thickness of the layers included in the thermal insulation system (acoustic insulation performance: $\mathrm{R}_{\mathrm{w}}=60 \mathrm{db}$ ).
Table 3: BIM object geometrical information for wall

\begin{tabular}{lc}
\hline Layers & $\begin{array}{c}\text { Thickness } \\
\text { [mm] }\end{array}$ \\
\hline Lime and cement plaster & 15 \\
Brick with pores & 250 \\
Lime and cement plaster & 15 \\
Weber.therm AP50 start & 8 \\
Isover clima34 G3 & 100 \\
Weber.therm TA8 & \\
Glass fibre reinforcement net & \\
Weber.therm AP50 start & 8 \\
Weber.prim RC14 & \\
Weber.cote Action F-R-M & 1.5 \\
\hline
\end{tabular}

As far as the roof and ground slab are concerned, the following solutions have been adopted and tested in the case studies (Table 4; Table 5). The thermal and acoustic performances of the roof have been provided by the BIM object, such as:

- total thickness: $355.5 \mathrm{~mm}$;

- thermal transmittance (declared): $0.2 \mathrm{~W} / \mathrm{m}^{2} \mathrm{~K}$;

- acoustic insulation performance: $\mathrm{R}_{\mathrm{w}}=42 \mathrm{db}$.
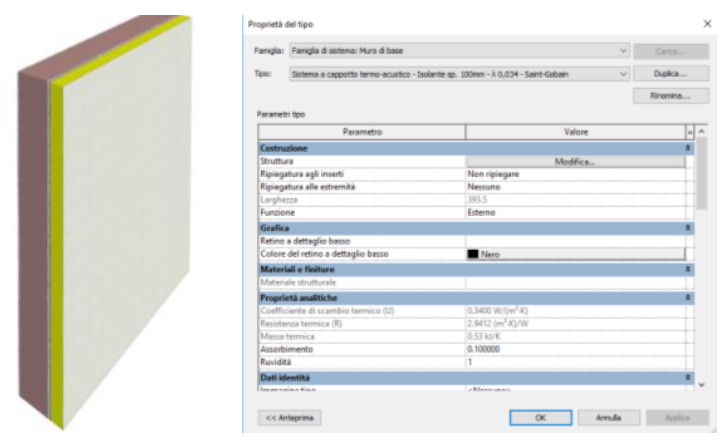

Figure 3: Type properties for the wall insulation system of the manufacturer

Regarding the ceiling system, the solution for energy retrofitting has been conceived as a rock wool insulation layer of Isover IBR K 4+:

- total thickness: $355 \mathrm{~mm}$;

- thermal transmittance (declared): $0.18 \mathrm{~W} / \mathrm{m}^{2} \mathrm{~K}$;

- acoustic insulation performance: $\mathrm{R}_{\mathrm{w}}=56 \mathrm{db}$ ).

\begin{tabular}{lc}
\multicolumn{2}{c}{ Table 4: BIM object of the roof } \\
\hline Layers & $\begin{array}{c}\text { Thickness } \\
{[\mathbf{m m}]}\end{array}$ \\
\hline Matchboarding & 20 \\
Isover VARIO KM DUPLEX & 0.8 \\
OSB panel & 19 \\
Wooden joists & 60 \\
Isover BAC CF N ROOFINE G3 & 60 \\
Wooden joists & 60 \\
Isover BAC CF N ROOFINE G3 & 60 \\
OSB panel & 19 \\
Air gap with joists & 50 \\
Bituver SYNTODEFENSE & 0.8 \\
Slats for tiles & \\
Tiles & 6 \\
\hline
\end{tabular}


Table 5: BIM object of the slab against ground

\begin{tabular}{lc}
\hline Layers & $\begin{array}{c}\text { Thickness } \\
\text { [mm] }\end{array}$ \\
\hline $\begin{array}{l}\text { Expanded clay concrete for unventilated } \\
\text { substrates }\end{array}$ & \\
Reinforced concrete & 200 \\
Overlap band Akustrip 33 & \\
Insulation Isover Ekosol N 4+ & \\
Bituminous cardboard Bituver Bitulan C3 & \\
Distribution screed & 40 \\
Ceramic tiles & 10 \\
\hline
\end{tabular}

\section{BIM object for the transparent envelope}

As far as the transparent envelope is concerned, the glazing systems have been defined throughout the glazed solution provided by the manufacturer's library as well as specific frames: the available BIM objects were only related to glasses, indeed. The glass-related BIM objects included the information about double and triple glazing systems without any additional details about the integration with the structural frame. The designer is then responsible for specifying the results involving the special analyses for the glazing systems (i.e., glass and frame, seals, glass stop, etc.). The manufacturer provides the object families: whenever the glass has to be included in the model, it is possible to check the possibilities given by the library and to replace the windows with the available objects (Figure 4). In the retrofitted situation, a double glazing system has been adopted due to the main market choices affected by economic reasons.

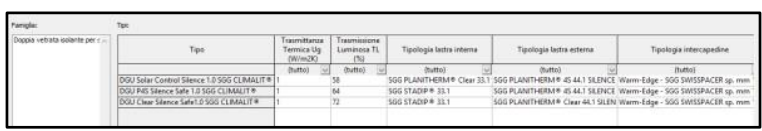

Figure 4: Manufacture's family for double glazing systems

Performance-related information that are needed in order to perform the energy analysis

The research aims at applying the BIM objects dealing with the thermal insulation system to the case studies (Figure 1) in order to evaluate how they can be used to support the energy analysis according to the energy parameters required by the national regulations (Italian Ministerial Decree, 2015). They require the calculation of specific thermal parameters needed to define the building energy performance (i.e., energy label) throughout a computational procedure. The procedure implements the standard building energy balance (UNI-TS 11300:2018). The energy calculation focused on the main parameters of thermal analysis in the static regime of the buildings as approved by the mentioned standard. A bi-directional link through VPL has been developed between the BIM models and an Excel spreadsheet, which implements the equations accomplishing the national standard calculation. The link has been developed in order to arrange and manage the computational procedure and to achieve the energy results for the case studies starting from the thermal information stored into the BIM objects included into the building information models. Data are then visualized in a dashboard displaying three information levels:

- climate data, related to the location of the existing building (UNI Standard 10349) including climate zone at territorial level, Degree Days (DD), Heating Days (Hdays);

- geometric data, about building typology, net area, gross volume, net volume, exposed to the outdoor surfaces, $\mathrm{S} / \mathrm{V}$ ratio (surface area exposed to the outdoor and gross heated volume ratio);

- performance data, referred to the thermal losses and gains, the acoustic performance of the evaluated building according to the parameters included in Table 6 .

Table 6 summarizes the thermal information and the main acoustic parameters, which derive from the layers included in the opaque envelope introduced into the BIM models through the manufacturer's BIM objects.

\begin{tabular}{|c|c|}
\hline Parameter & Unit \\
\hline $\begin{array}{l}\text { Thermal transmittance } \\
\text { - } \text { external wall } \\
\text { - } \text { roof } \\
\text { - } \text { slab } \\
\text { - } \text { windows }\end{array}$ & $\mathrm{W} / \mathrm{m}^{2} \mathrm{~K}$ \\
\hline $\begin{array}{l}\text { Solar contributions through windows } \\
\text { Solar collection area }\end{array}$ & $\begin{array}{c}\mathrm{kWh} \\
\mathrm{m}^{2}\end{array}$ \\
\hline Acoustic insulation performance & $\mathrm{db}$ \\
\hline $\begin{array}{l}\text { Stationary heat exchange } \\
\text { - to outside } \\
\text { - to ground } \\
\text { - to unheated spaces }\end{array}$ & $\mathrm{W} / \mathrm{K}$ \\
\hline $\begin{array}{l}\text { Energy demand } \\
\text { Energy performance in winter period }\end{array}$ & $\begin{array}{c}\mathrm{kWh} \\
\mathrm{kWh} / \mathrm{m}^{2} \mathrm{y} \\
\mathrm{kWh} / \mathrm{m}^{3} \mathrm{y}\end{array}$ \\
\hline Energy label & A4-G \\
\hline
\end{tabular}

The findings and outcomes stemming from the BIM objects' analysis concerning the ETICS, as well as the predefined Revit objects, show how the information included needs an integration before starting the data transfer procedure in order to fill correctly any spreadsheet cells required for performing the energy analysis. A customized digital representation of the building components had to be developed, including user-defined properties.

For instance, the thermal transmittance, associated to the windows and related to the glass surface and the frame specific features, has been added to the "window" BIM object as well as its area, which is requested in order to calculate the heat gains given by the solar radiation through the windows; the solar collection area should be added modifying the window family, too (Figure 5). 
The data enrichment procedure is a crucial step for BIM modeling in order to assure a reliable level of information need based on the relevant BIM use. It is strongly related to the final task of the data export. Some needed data for the energy calculation are related to the materials' properties, while others to the dimensions of surfaces; therefore, in some cases, it is possible to get the information as a direct output, while for other situations special equations have to be implemented in the BIM object's properties in order to obtain the combined data as, for example, the area given by the dimensions of the windows (i.e., length and width) (Figure 5).

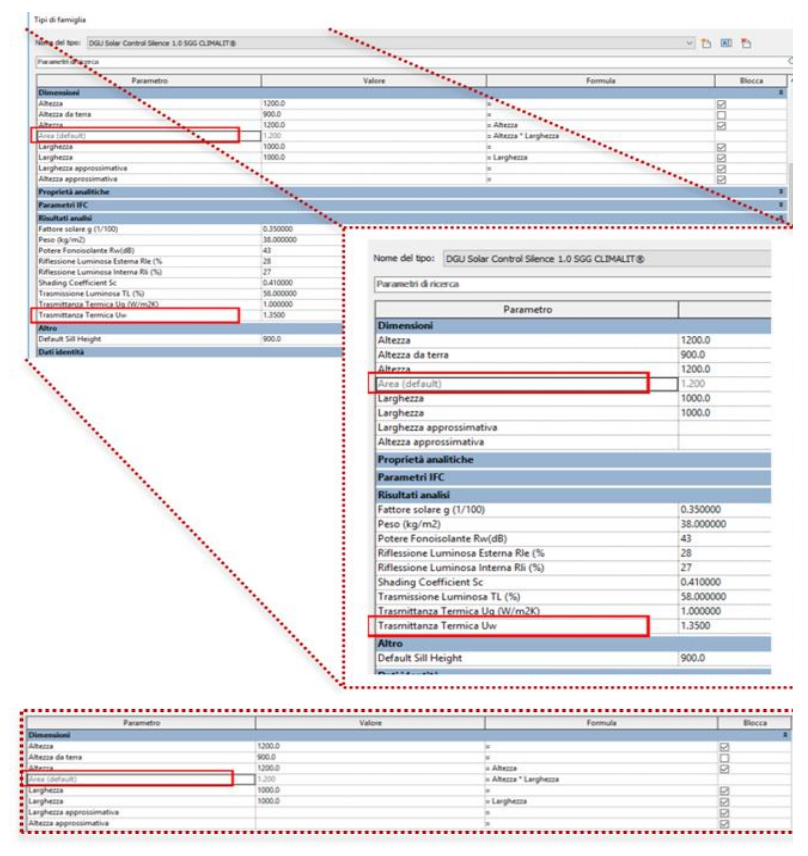

Figure 5: Family of windows available in the manufacturer's website modified to include the values needed for the energy evaluation

\section{Definition of the calculation model for energy performance assessment}

The calculation model has been developed in Excel organizing different sheets for each specific analysis referred to the national regulation requirements. which imply component and building energy performance levels. The spreadsheet has $n$. 13 sections arranged to cover thermal and acoustic requirements. The list of the calculation sections is as follows:

1. Dashboard: the summary of the performance data is displayed for each implemented case study (Table 6);

2. $Q h$ : the energy label is calculated as heating demand of the whole building;

3. HD: the stationary specific thermal losses of the opaque vertical, horizontal envelope and of the transparent surfaces is calculated based on area of the component and thermal transmittance of the opaque and transparent components;

4. $\mathrm{Hg}$ : the stationary specific thermal losses to ground based on area of the component and thermal transmittance of the slab is calculated;

5. $H U$ : the stationary specific thermal losses to unheated spaces based area of the component and thermal transmittance of the components is calculated;

6. Qs: solar gains through windows based on solar radiation of the climate zone, orientation of the window and size of the window are calculated;

7. Asol: the solar collection area is calculated

8. $D 2 m, n T, W$ : the acoustic insulation effect of the components is calculated;

9. Uwall: it is possible to organize the stratification of the vertical opaque envelope components based on custom materials and on the BIM library components and a comparison with the limit of thermal transmittance provided by law is automatically performed.

10. Uroof: it is possible to organize the stratification of the horizontal opaque envelope (roof) components based on custom materials and on the BIM library components and a comparison with the limit of thermal transmittance provided by law is automatically performed;

11. Uslab: it is possible to organize the stratification of the horizontal (slabs) opaque envelope components based on custom materials and on the BIM library components and a comparison with the limit of thermal transmittance provided by law is automatically performed.;

12. Uwindows: the thermal transmittance calculation of the windows;

13. Model data: surface area and thermal transmittance of the components of the building are transferred by the BIM model.

The sections of the spreadsheet are correlated and connected to realize the calculation starting from the BIM data and visualizing the results in the Dashboard section.

\section{The bi-directional data transfer link from the BIM} model to the energy calculation spreadsheet

The information provided by the building information model, including BIM objects that have been enriched with user-defined properties, have been transferred to the Excel spreadsheet implemented with the equation for the energy balance included in the national regulation through the VPL (Visual Programming Language); the input data have been used to calculate the predefined performance parameters. In this way, it was possible to enable the efficient use of the BIM library through the application of product selection procedures and performance calculations based on a functional workflow from the information model to the Excel spreadsheet. Autodesk Dynamo has been used to link the building information model to the Excel spreadsheet where n. 13 sections have been arranged as described in the previous section. In particular, in 
Autodesk Dynamo VPL can be used to transfer data from Autodesk Revit to Excel and vice versa. In this study, the thermo-physical information has been used to populate the calculation model in Excel in order to evaluate the possible energy upgrade of the case studies throughout the BIM library system adopted in the building information models. The adopted tools are shown in Figure 6.

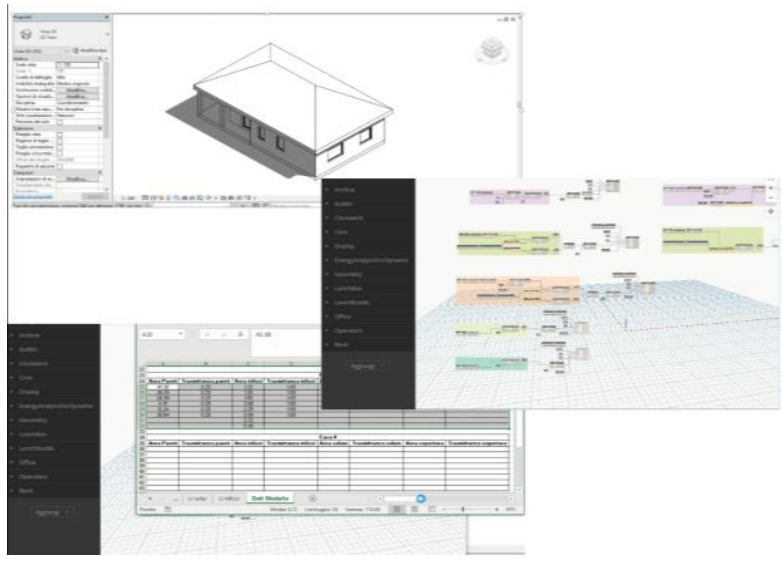

Figure 6: Workflow of data transfer from the building information model to the calculation spreadsheet through VPL

The building information models have been conceived as data sources for the energy calculation spreadsheet, considering the main parameters needed for the geometrical and alpha-numerical data sections. The idea is that the BIM object can be used by the practitioners in their project where refurbishment envelope solutions are required. The use of the BIM object with the data enrichment provide the data to the calculation tool through the transfer link giving the feedback about the achievable performance with the BIM configuration. The bi-directional link is used to communicate to the BIM environment the energy quality of the solution and thus compare different options to reach the energy goal. The procedure requires the filtering of any object classes by categories. In this way it is possible to select all the elements of the same category within the BIM model and then to sort them by name (Elements.FilterByName) in order to obtain the list of the specific components of interest. Based on the list, it is then possible to select the specific properties that have to be exported in order to fill the relevant cells in the spreadsheet for enabling the energy analysis (Element. GetParameterValueByName).

The same procedure has been applied also to the windows using the specific category. Through the BIM object (Family Types) it is possible to write down the complete list of the family features (Get Family Parameters). For the roof and slab components the type properties have been then selected through the element name (RoofType.ByName) (Figure 7).
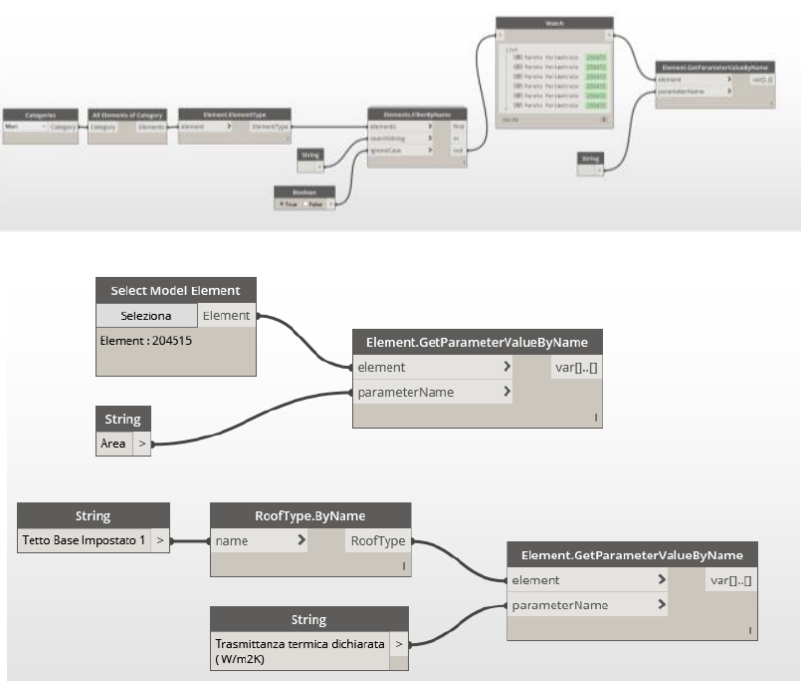

Figure 7: Dynamo script for wall data transfer and roof performance data transfer (as example the declared thermal transmittance)

In order to enhance the data transfer process, it is crucial to realize the correct link in the VPL understanding if the needed information is related to the type properties or to the family properties. In fact, the structure of the script has to be customized in the different situations for the data extraction and communication to the calculation model in Excel.

\section{Results of the energy analysis}

The calculation of the energy retro in the three case studies shows the following results:

- in building (a), the single-family house the vertical walls insulation assures an energy saving of about $27 \%$. The windows' replacement (with a double glazing system) and the roof insulation lead to an average $78 \%$ of energy saving for space heating. The insulation of the slab reduces of $6 \%$ the previous results (Figure 8).

- For building (b) residential apartments, the wall insulation can improve the energy performance in Milan of $17 \%$, in Rome of $19 \%$ and in Palermo of $23 \%$. The higher implementation is achieved with windows' replacement and roof insulation with $67 \%$ of energy saving in Milan, $76 \%$ in Rome and $88 \%$ in Palermo. The insulation of the slab increases the performance of about $16 \%$.

- For the office building (c) the evaluation has been defined applying all the energy retrofit measures at the same time and a whole reduction of $61 \%$ of the energy demand could be achieved in Milan, a $69 \%$ of energy saving has been registered in Rome and an $80 \%$ could be achieved in Palermo (Figure 9). 


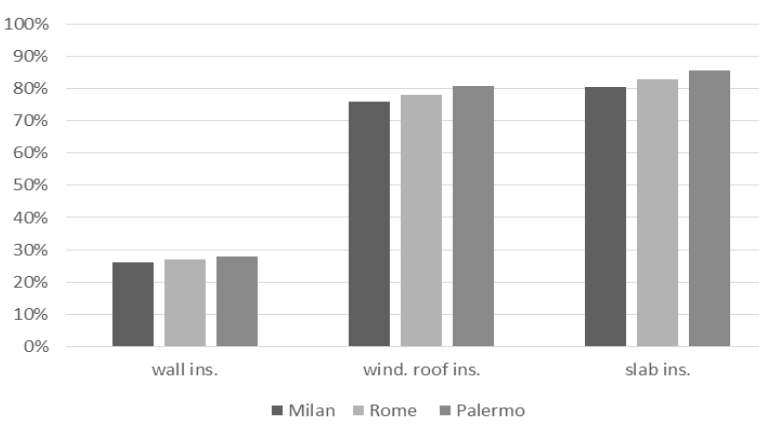

Figure 8: Performance implementation with retrofit measures in the three climate for the building (a)

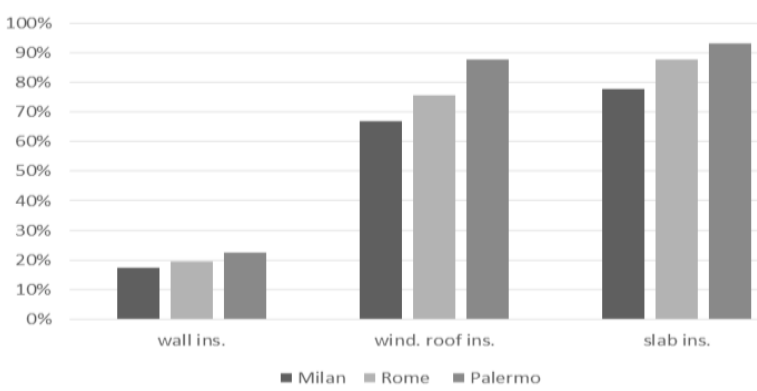

Figure 9: Performance implementation with retrofit measures in the three climate for the building $(b)$

\section{A recommender for BIM object-based design of buildings}

According to the research perspective, BIM object libraries perform as the core components of a novel web application that has been developed in order to support design teams in using libraries in a more advanced and fruitful way. In particular, this application is meant (i) to implement energy analysesoriented services on selected BIM objects, as described in the previous section, and (ii) recommendations for the BIM objects suitable for being integrated into a given design, as discussed in this section. The web application relies on a conceptual data model aimed to describe the relevant classes of data and associations, represented by means of a UML class diagram. The model focuses on describing the use of BIM objects in the design process. Figure 8 reports the resulting diagram in terms of classes, associations, and related multiplicities, where attributes are not detailed for the sake of compactness of the diagram. This model defines the data persistence component (i.e., the database) of the application. From an operational point of view, designers have to register to the web application by specifying their demographic data and business profiles. Then, they may describe one or more building designs in terms of, among the others, a description of the context (e.g., the building type and its location), applicable energy class, and, for each design the used BIM objects. After entering this data, designers are enabled to gain benefits from the services provided by the application. For instance, designers can ask for an energy performance analysis to be performed on a targeted building. Moreover, they can get a recommendation consisting of a ranked list of BIM objects as alternatives to a currently selected one to be used in the target building.

In particular, recommended objects are selected according to a collaborative recommendation approach that relies on information such as features of the target building, its context, and past selections of BIM objects performed by the current and by other designers. The data model in Figure 10 can be shortly described in terms of the following requirements. Conventionally, in the following description, the authors capitalize the first letter of names of classes appearing in the diagram. A User of our web application registers to Data sources providing (libraries of) BIM objects to be used as components in Designs. A Design is associated with a Context defined by a Location, and a Type of building. Hence, an Analysis model (e.g., energy performance evaluation) can be adapted to a specific Context to keep it into account. A BIM object has a list of Performance parameters representing measures of precise technological aspects related to the object composition. A BIM object may be associated by the user with Alternative objects, which are BIM objects suitable for replacing the object thanks to, for example, having similar features even if they are produced by a different company. Moreover, an object belongs to an object Category. A User may define a BIM object variant in order to store the description of a customization she/he made to a predefined BIM object. Finally, Analysis models provide relevant evaluations applicable to both specific BIM objects and objects Categories. The authors are now going to discuss the configuration of the recommendation service for our web application, based on the presented data model. The recommender is designed to deliver a ranked, i.e., ordered by relevance, list of objects given a request expressed by a designer. From a technical point of view, the algorithm implementing the recommender combines a content-based selection of objects to be recommended with a collaboration-based ranking of them. The algorithm uses data available in a database coherent with the model in Figure 10.

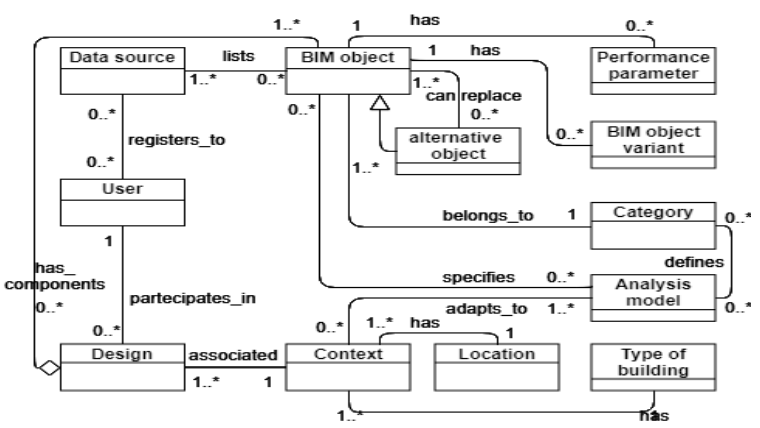

Figure 10: Conceptual data model design for the application 
A recommendation request is specified by the following data:

- either an object category describing the desired category of object $(C)$, or (ii) a specific object $(o)$ for which alternative objects are searched;

- parameters of the target design $D$ : type of building (D.Context.Type_of_bdng), average cost (D.avg_cost), degree-days (DD) of the building location (D.Context.Location.DD), energy class $(D . E C)$;

- intended use $I U$ of the recommended objects.

This means the designer specifies an intended use for the BIM objects to be recommended, for example "energy retrofitting". An intended use $I U$ identifies a minimum set of attributes required IU.attr. In our proposal, for the energy retrofitting intended use, the set IU.attr is consistent with the minimum set of data required as input by the energy performance calculation model described in the previous section.

Then, a recommendation request is processed in the following way (Figure 11). Firstly, all the available past designs similar to the design $D$ are retrieved from the database at phase 2) according to a content-based approach. Here, an overall similarity between a pair of designs is defined and, in our proposal, this means comparing primary and contextual features of the buildings subject of designs. Formally, the overall similarity is established by four conditions based on simple similarity criteria: 2.1 ) the two buildings should be of the same type; 2.2) they should have close average cost per unit of surface/volume; 2.3) they should be at locations with close values of DD; 2.4 ) they should have close, or the same, energy classes.

Preliminarily, at phase 1), the maximum admitted ranges for the similarity criteria are set (e.g., the maximum difference for DD). These ranges (e.g., $\alpha, \beta$, and $\gamma$ ) will be set to optimal values after performing wide experimentation with the application and users. As well as, at this phase, the category $\mathrm{C}$, if not provided, is set to the one of the object $o$.

Then, all objects used in the designs similar to $D$, denoted as $S D$ and selected by the steps at phase 2), are retrieved at phase 3). These objects are the candidates to be recommended.

In the next phase 4), these candidates are filtered out, firstly, by removing the ones not belonging to the category $C$ requested by the user, as specified by the category-based filtering phase 4.1). Secondly, by removing the candidates having a set of descriptive attributes that do not include the minimum set of attributes required for the intended use of the object, as reported in phase 4.2). Finally, in the last phase 5) each object in the filtered candidate objects $C O$ is ranked proportionally to the number of designs that are similar to $D$, and use this object. This means that most used objects are presented better than less used ones.
The ordered list of objects is shown to the user.
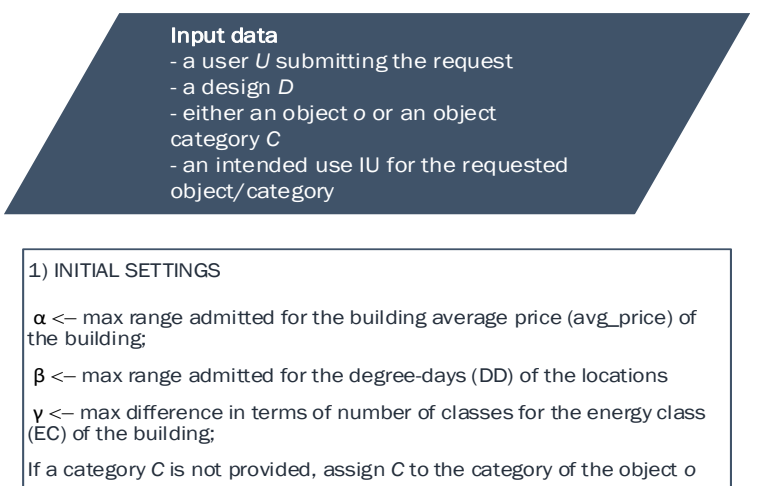

4) CATEGORY-BASED AND USE-BASED FILTERING OF OBJECTS

4.1) Remove from $C O$ the objects o not belonging to the category $C$

4.2) Remove from $\mathrm{CO}$ the objects o with attributes o.attr not inclusing the minimum set of attributes IU.attr for the intended use

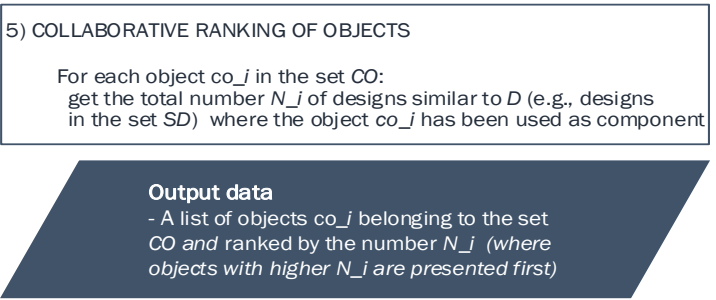

Figure 11: Description of the phases and steps of the recommender

In order to preliminary evaluate the proposed approach, output of the proposed recommender could be compared to the output of other approaches, i.e., (i) keyword-based search systems operating on textual descriptions associated to BIM objects, (ii) general purpose recommender systems configured to work on BIM objects, (iii) faceted search systems permitting to filter out the objects in one or more libraries based on filtering criteria expressed by designers. The authors assume all these systems, including ours, provide outputs as top-k lists, based on proposing as output of the system the best k matching objects. Based on this realistic assumption, the authors are planning to compare ours to other approaches based on both (i) 
classical information retrieval measures (e.g. precision) involving a user-centered evaluation of the produced output, and (ii) specific top-k distance metrics (e.g., generalized Kendall's Tau measure).

\section{Conclusions}

The available BIM objects either as predefined in BIM authoring tools or as user-defined objects by the manufacturer usually do not include the appropriate informative content for the energy analysis purposes. The BIM-to-BEM interoperable workflow remains an open issue for effective design integration. The study represents a preliminary attempt to support the development of an energy-analysis procedure focused upon BIM objects.

This study analyses the use and customization of a manufacturers' BIM library for energy retrofitting purposes. The library is available on a digital marketplace and the users can access and download it for free. Anyway, the BIM objects developed by the manufacturers do not allow an automatic BIM-toBEM analysis workflow and, consequently, some customization is needed. For that reason, the paper has adopted some case studies as a method to identify the information requirements needed to perform a preliminary energy analysis for retrofitting purposes during the design phase. Based on this results, the study also discusses the design of a software system for recommending the most appropriate BIM object to be used for the specific purpose and use case based on the previous experiences of other users, in order to guide the selection of the BIM object in future projects.

Such a system could support organizations and designers in the selection of the most suitable object to create their own BIM models based on previously defined purposes and use cases, acquiring knowledge from experience. Being a first step into the development of the web system, future works are needed for (1) completing its design both for energy analysis purposes and other use cases for which a recommender system could be used for; (2) developing the first prototype version of the system; (3) testing the systems on multiple projects and configurations in order to evaluate its effectiveness.

\section{Acknowledgments}

The authors want to acknowledge Saint-Gobain Italia and Weber for their availability and useful discussion about the performance parameters in the setup phase. Special thanks go to the graduate student Alessio Mazzotti for the crucial contribution during the modeling and analysis phases.

\section{References}

Aggarwal, C.C., (2016) An introduction to recommender systems. In: Recommender Systems, pp. 1-28, Springer, Cham.
Burke, R. (2007) Hybrid web recommender systems. In: The adaptive web, pp. 377-408, Springer, Berlin, Heidelberg.

Eastman, C., Teicholz, P., Sacks, R., \& Liston, K. (2011). BIM handbook: A guide to building information modeling for owners, managers, designers, engineers and contractors. John Wiley \& Sons.

Gourlis, G., \& Kovacic, I. (2017) Building Information Modeling for analysis of energy efficient industrial buildings. A case study. Renewable and Sustainable Energy Reviews, 68, pp. 953-963.

Hjelseth, E. (2010) Exchange of relevant information in BIM objects defined by the role-and life-cycle information model. Architectural Engineering and Design Management, 6(4), pp. 279-287.

Italian Ministerial Decree (DM 26/06/2015) Minimum Requirements for the Energy Performance of Buildings.

Kim, J. B., Jeong, W., Clayton, M. J., Haberl, J. S., \& Yan, W. (2015) Developing a physical BIM library for building thermal energy simulation. Automation in construction, 50, pp. 16-28.

Kreider, R. G., \& Messner, J. I. (2013) The uses of BIM. Classifying and Selecting BIM, Pennsylvania State University (9th version).

Ricci, F., Rokach, L., \& Shapira, B. (2015) Recommender systems: introduction and challenges. In: Recommender systems handbook, pp. 1-34, Springer, Boston, MA.

Sacks, R., Eastman, C., Lee, G., \& Teicholz, P. (2018) BIM Handbook: A Guide to Building Information Modeling for Owners, Designers, Engineers, Contractors, and Facility Managers, John Wiley \& Sons.

UNI 10349-1:2016 Heating and cooling of buildings Climatic data - Part 1: Monthly means for evaluation of energy need for space heating and cooling and methods for splitting global solar irradiance into the direct and diffuse parts and for calculate the solar irradiance on tilted planes.

UNI 10349-3:2016 Heating and cooling of buildings Climatic data - Part 3: Accumulated temperature differences (degree-days) and other indices

UNI/TS 11300-1:2018 Prestazioni energetiche degli edifici - Parte 1: Determinazione del fabbisogno di energia termica dell'edificio per la climatizzazione estiva ed invernale. 